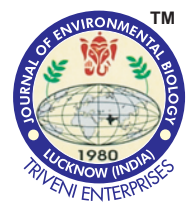

\title{
Production of antimicrobial adhesives
}

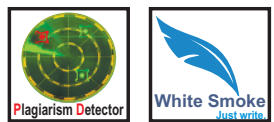

\section{Authors Info}

D. Yuvaraj*, R. Gnanasekaran, J. Iyyappan, I. Subashini, S. Nandhini, M. Jayasudha, R. Shaleni and M. Shyam Department of Biotechnology, Vel Tech High Tech Dr. Rangarajan Dr. Sakunthala Engineering College, Avadi, Chennai-600 062, India

*Corresponding Author Email : yuvarajdinakarkumar@gmail.com

\section{Edited by \\ Professor M. Seenuvasan}

\section{Reviewed by}

Dr. J. Madhusudhanan

Dr. N. Balaji

\begin{abstract}
Aim: To produce a bio mimetic consortium embedded with chitosan, haemolymph and calcium nanoparticles to enhance its activity in wound healing.

Methodology: Haemolymph was collected with a syringe from the abdominal region of the snails. Nanoparticles were prepared from the powdered samples of shells and operculum by addition of $\mathrm{HCl}$ and $\mathrm{NaOH}$. Later, it was calcinated in the muffle furnace. SEM analysis was performed to check for the formation of nanoparticles. To extract chitosan, the cockroaches were dried in hot air oven, then crushed and powdered. By the process of de-protenization and de-mineralization, chitin was obtained by de-acetylation process to produce chitosan. Haemolymph was checked for anti-bacterial property which was determined by agar dilution method.
\end{abstract}

Results: The results obtained indicated that the consortium prepared from the combination of calcium nanoparticles, chitosan and haemolymph proved to have an impact on the growth of Escherichia coli, Pseudomonas aeruginosa, Bacillus subtilis, Bacillus licheniformis. These synthesized compounds has individual potency too.

Interpretation: All the samples, haemolymph, calcium nanoparticles and chitosan showed anti-microbial property and were embedded in the bandages. Hence, the wound healing property of bandages were increased and its activity was verified.

Key words: Antibacterial activity, Bandages, Calcium nanoparticles, Chitosan, Haemolymph.

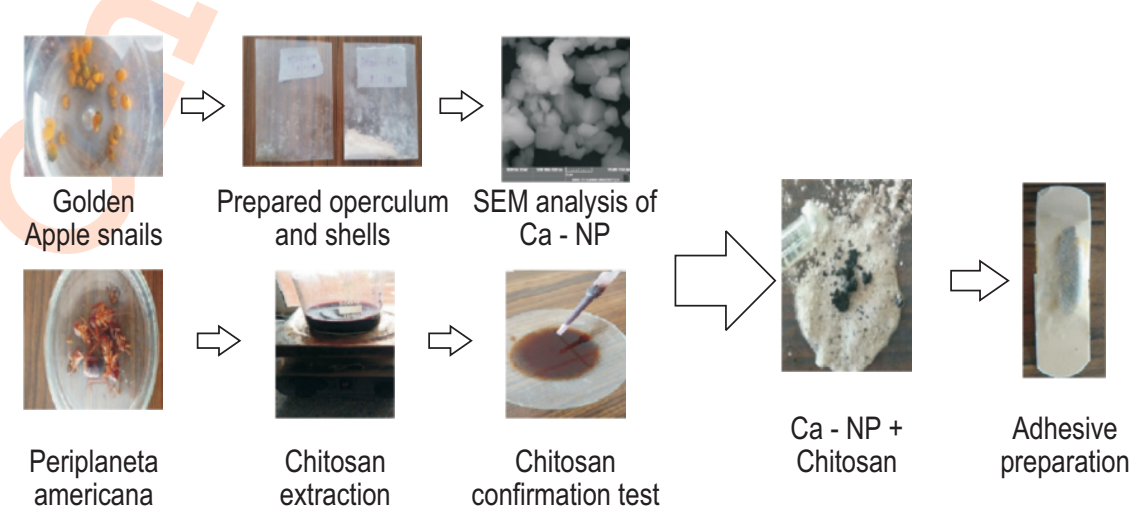

How to cite : Yuvaraj, D., R. Gnanasekaran, J. Iyyappan, I. Subashini, S. Nandhini, M. Jayasudha, R. Shaleni and M. Shyam: Production of anti-microbial adhesives. J. Environ. Biol., 40, 812-816 (2019). DOI : http://doi.org/10.22438/jeb/40/4(SI)/JEB_26 


\section{Introduction}

Injury of any kind caused to the epidermis, local vasculature and the underlying tissues creates a potential threat to the integrity of the organism as it can allow the entry of pathogens, as well as blood loss (Daunton et al., 2012). Wound healing concludes with up to $80 \%$ original tensile strength being restored to the site. It involves four tightly overlapping phases: Hemostasis (formation of fibrin clot); Inflammation (body's immune response); Proliferation (cell migration and wound closing) and Remodeling (reorganization of the disorganized matrix to uninjured tissue).

The use of gauze and bandage to dress wound plays an essential role in health care sectors. It ranges from simple dressing to superficial wounds to holding together fractured bones or body parts from rehabilitation and recovery. They are mainly used in wound healing mechanism.

The material are of two types: Woven gauze - cotton cloth and Synthetic gauze - rayon or synthetic materials (Daunton et al., 2012).Current wound dressings have disadvantages like poor flexibility, poor mechanical strength, lack of porosity, and a tendency for dressings to adhere onto the wound surface (Jayakumar et al., 2011). It is well known that bacterial adhesion is the first step in colonization of wounded skin and in formation of a bio-film. Therefore, it is very important to have the antibacterial surface directly on to the bandage to avoid the formation of these bacteria films, preventing bacterial multiplication inside the bandage (Juknius et al., 2016). Bio-materials can assist in proper physiological reconstruction of skin and reduce or prevent scar tissue formation. Some of them are chitin, chitosan, silver, iodine, calcium, etc. (Azad et al., 2004). In large open wounds, the process of new tissue formation is recognized as granulation, which should form before wound epithelialization begins. Therefore, chitosan has an adaptable property for the wound (Ueno et al., 2001). Materials for wound dressing and tissue engineering are important but still under development. New adhesives have been proposed. Chitosan derivative is non-toxic, cyto-compatible and mechanically suitable for peripheral surgeries. The poor solubility of chitin is the major limiting factor in its utilization and investigation of its properties and structure. Despite these limitations, various applications of chitin and modified chitins have been reported as raw materials for absorbable sutures and wound dressing (Dutta et al., 2004).

Mollusca innate immunity relies on both cellular and humoral component. Cellular components comprehends, among the most important protagonists, circulating cells able to engulf, encapsulate or simply kill the invaders and potential pathogens. These cells are usually called hemocytes, and despite the considerable efforts spent for their characterization, their origin and classification still remain to be firmly established in mollusks (Accorsi et al., 2013). Three circulating hemocyte types were distinguished such as hyalinocytes, agranulocytes and granulocytes (Cueto et al., 2011). Cockroaches are used in Traditional Chinese Medicine (TCM) against bone tuberculosis, cirrhosis, liver cancer, breast cancer and as facial beauty mask because of their purported anti-aging effects. Chitin is especially important in the extracellular structures and is available biopolymer which is used in various industrial and pharmaceutical applications. Chemical composition of chitin is $\beta$ - (1-4) 2 acetamido-2-deoxy- $\beta$ - $\mathrm{D}$-glucose ( $\mathrm{N}$-acetyl glucosamine) and the deacetylated derivatives of chitin are chitosan which is a linear polymer of a (1-4 linked-2- amino-2-deoxy- $\beta$-D-glucopyranose). These have specific applications in drug delivery, tissue engineering, food sectors, waste water treatments, imprinting and metal nano-composites (Kaewboonruang et al., 2016).

Snails are preferably available and does not cause any harmful effects to humans. In countries like China, Japan and Europe cockroaches are consumed. Insects can play a vital roles in the transmission of pathogenic bacteria and fungi with antibiotic resistance in households and in hospital environments (Salehzadeh et al., 2007).

The present study aimed to produce bandages embedded with chitosan, haemolymph and calcium nanoparticles to enhance its activity in wound healing.

\section{Materials and Methods}

The consortium was developed from a mixture using the operculum and shell of Pomacea canaliculata: Common name Golden apple snail. Family- Ampullariidae and the haemolymph of Periplaneta americana: Common name - Cockroaches Family - Blattidae.

Snails and cockroaches were collected from different localities in Chennai. The snails were allowed to grow with proper aeration and regular feeding. The haemolymph was collected the abdominal region. The collected samples was centrifuged at 3000 rpm for about 3 times and EDTA was added to the supernatant and stored in refrigerator for further use. It is well known that haemolymph has anti-microbial activity and it was tested by using agar dilution method in subsequent steps. The snail shell and operculum was removed from its body after euthanizing the animals. The haemolymph was collected from the cockroaches by a syringe near the base of their legs. Both the samples were dried in the hot air oven at $65^{\circ} \mathrm{C}$ for about 3 days. The samples were crushed separately with a mortar and pestle to make a fine powder.

Anti-bacterial assay was confirmed with the collected haemolymph by using four different bacterial samples by agar dilution method. Agar was selected as a medium for growth of microorganisms and the culture was streaked with L-rod. Haemolymph was poured in the centre of Petriplate by gel 


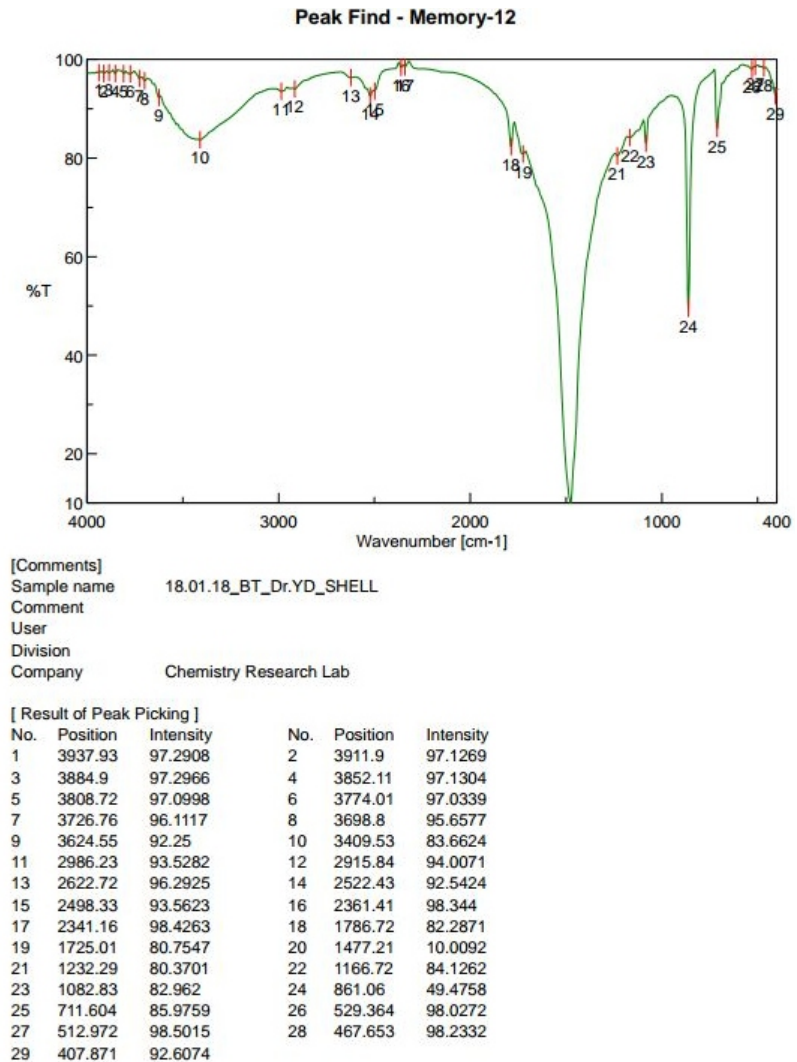

Fig. 2 : Graph of FTIR analysis of the snail shell

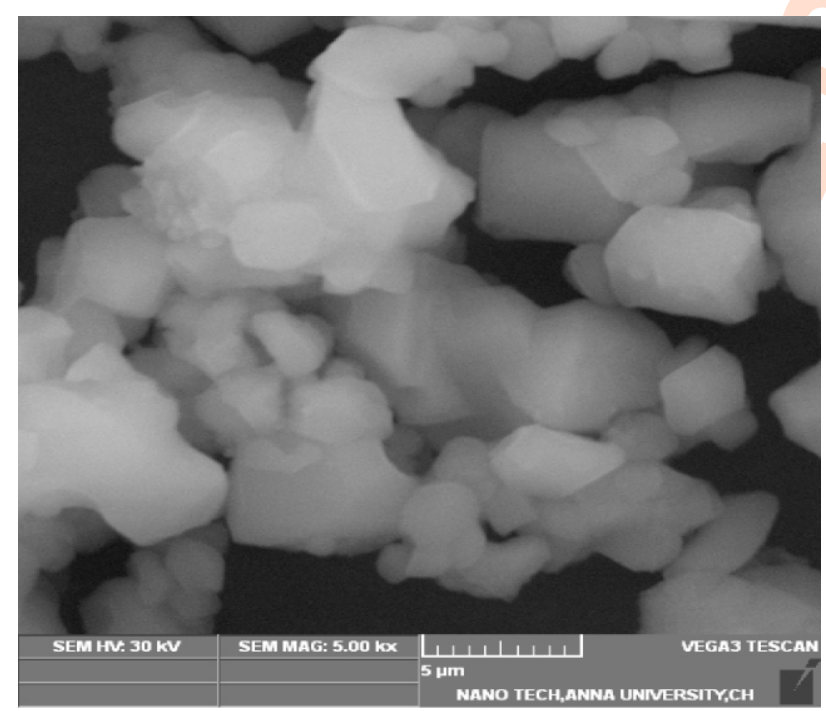

Fig. 4 (a) : SEM analysis of calcium nanoparticles

punching method. It was incubated for about 48 hours and the plates were checked for the microbial growth.

Calcium nanoparticles were prepared from the shells and the operculum of the snail. Nanoparticles was prepared by
Peak Find - Memory-14

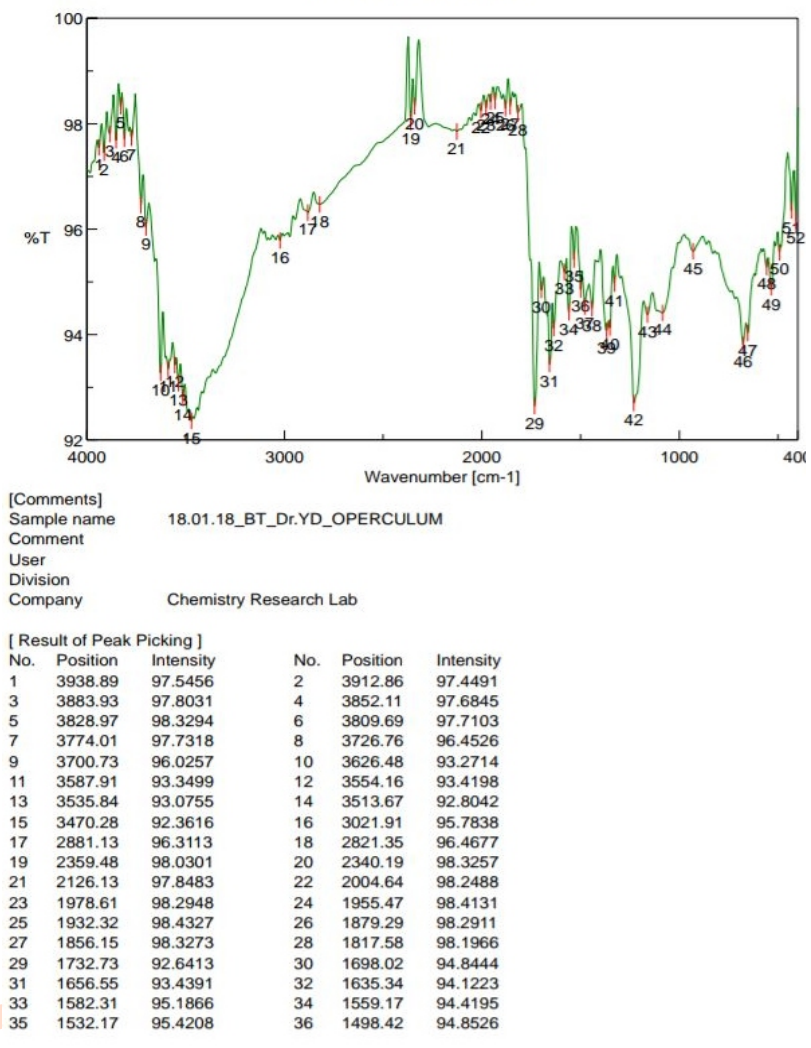

Fig. 3 : Graph of FTIR analysis of the operculum in the snail

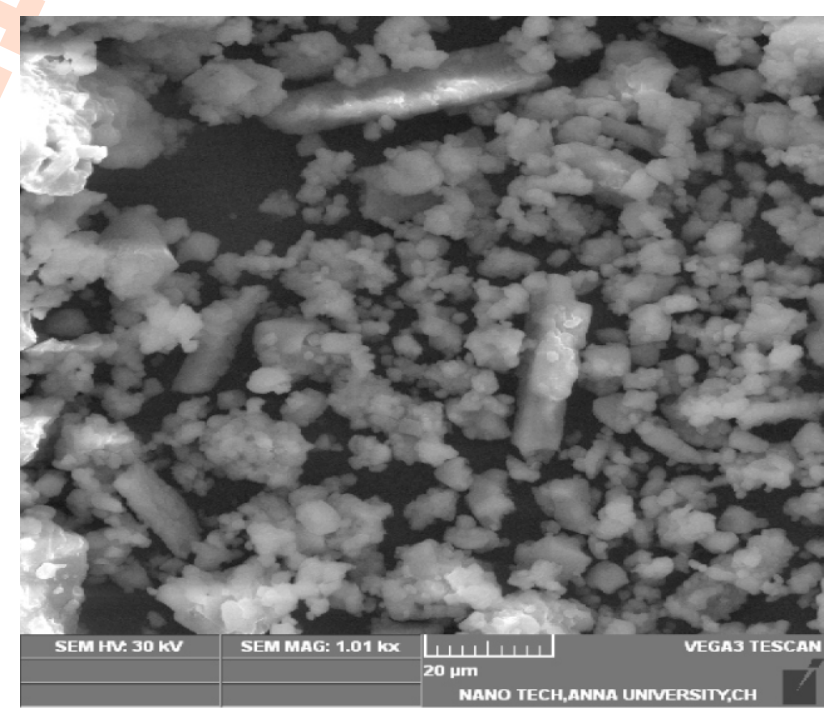

Fig. 4 (b) : A result of SEM analysis

precipitation method (Abdul et al., 2013, Ferraz and Monteiro, 2004, Teerawat, 2014, Himanshu and Satya, 2016) 2-5. 1M calcium nitrate and $0.6 \mathrm{M}$ Di-ammonium hydrogen phosphates was dissolved in distilled water and adjust the $\mathrm{pH} 10-11$ by adding ammonia with cationic surfactan $\mathrm{CTAB}$. The precipitate was 
obtained after washing with distilled water and ethanol. Finally Nano particles were rinsed with distilled water and dried in an oven at $80^{\circ} \mathrm{C}$ for $12 \mathrm{hrs}$ and then powder was crushed and kept at $800^{\circ} \mathrm{C}$ in a muffle furnace for $2 \mathrm{hrs}$. The confirmation of nanoparticles were done using FTIR and SEM analysis.

\section{Results and Discussion}

The collected haemolymph showed resistance against all the bacterial samples namely Escherichia coli, Pseudomonas aeruginosa, Bacillus subtilis and Bacillus licheniformis cultured in our laboratory. Pavlina et al., (2011) states that the snails have a complex mixture of biochemically and pharmacologically active components. It can be used in the medicinal field and also as an antibiotic.

The nanoparticles synthesized from the operculum and shell of golden apple snail was subjected to FTIR analysis to confirm the function groups (Fig. 2) and SEM analysis to confirm their size (Fig. 4 a, b).

The characteristic peaks of $\mathrm{C}-\mathrm{O}$ stretching and bending modes of $\mathrm{CaCO} 3$ are observed at $2522 \mathrm{~cm}^{-1}, 1477 \mathrm{~cm}^{-1}, 861 \mathrm{~cm}^{-1}$ and $467 \mathrm{~cm}^{-1}$. The observed peaks are compared with the standard graph to confirm its contents. The peak of the operculum is shown in (Fig.3).

By comparing the two results obtained from the operculum and the shell, calcium peaks are confirmed in both the shell and the operculum.

Ongluki et al. (2012) had discussed his FTIR results of the Golden apple snail and the inference of the peaks. This was used as a standard graph for the sample. Hence, the comparative study was useful in adding the operculum powder to prepare nanoparticles. Vanitha et al, 2017, obtained strong bands at 640 and $3702 \mathrm{~cm}^{-1}$ indicate the presence of hydroxyl groups in hydroxy apatite. The band at $1976 \mathrm{~cm}^{-1}$ is the presence $\mathrm{CO}_{3}{ }^{2-}$ groups.

The SEM studies indicated that the calcium particles obtained slightly exceeded the size of nanoparticles but were able to mix along with the other ingredients during the preparation of the adhesives (Fig. 4 a, b)

Nanoparticles are known to have anti-bacterial activity; hence the obtained particles were tested for their antibacterial efficacy. These nanoparticles have efficiency against Escherichia coli, Pseudomonas aeruginosa, Bacillus subtilis and Bacillus licheniformis. The nanoparticles were deposited at the centre by gel punching. After $48 \mathrm{hrs}$ of incubation, the plates were checked for its zone of inhibition. As they showed effective inhibition against all the four bacterial speces, it was evident that the calcium nanoparticles are efficient in wound healing activity against microbes. (Ataee et al., 2011) reported that the calcium nanoparticles have anti-bacterial effect on Gram positive and Gram negative bacteria. This was performed by inoculating both Gram positive and Gram negative bacteria and incubation was carried out for $48 \mathrm{hr}$ and the result obtained was positive.

Chitosan extracted by crushing whole cockroach was tested for confirmation. There was a change in colour of the solution during the addition of iodine followed by a colour change to dark purple when $\mathrm{HCl}$ was added. Wanule et al., (2017) suggested the confirmatory test for the chitosan. It states that the colour change is the best confirmatory test for the chitosan solution. The change of colour from yellow to dark purple indicates the presence of chitosan. The synthesized chitosan can be embedded in hydrogel to be used for wound healing (Wang et al., 2017). Chitosan is supposed to act as drug carriers as well (Jose et al., 2016).

Haemolymph, calcium nanoparticles and chitosan have anti-bacterial activity and it enhances the wound healing property. All the prepared particles were mixed together and the moisture content was removed by drying. Then it was lyophilized and stored. Later, it was embedded in the cotton area of the bandages and sealed. This adhesive would help both in the absorption and also wound healing activity.

\section{References}

Abdul-Rauf, I., W. Wei, D. Zhang, H. Wang and J. Li: Conversion of waste eggshells to mesoporous hydroxyapatite nanoparticles with high surface area. Materials Letters, 110, 195-197 (2013).

Accorsi, A., L. Bucci, M. de Eguileor, E. Ottaviani and D. Malagoli: Comparitive analysis of circulating Hemocytes of the freshwater snail Pomacea canaliculata. Fish. Shellfish Immunol., 34, 1-9 (2013).

Ataee R.A, P. J. Derakhshan, T. A. Mehrabi and A. Eydi: Antibacterial effect of calcium carbonate nanoparticles. Iranian J. Military Medi., 13, 65-70 (2011)

Azad, A. K., N. Sermsintham, S. Chandrkrachang, W. F. Stevens: Chitosan membrane as a wound-healing dressing. Characterization and clinical application. J. Biomed. Mater. Res. B. Appl. Biomater., 69, 216 -222 (2004).

Carmen Gonzalez and GeoffAllan: Preparing feed. NSW - Department of Primary Industries. ISBN 9780734718020 . (2007).

Cueto, J. A., M. Giraud-Billoud, I. A. Vega and A. Castro-Vazquez: Haemolymph Plasma Constituents of the Invasive Snail Pomacea canaliculata. Malacol. Soci. Austra. Soci. Study Mollus. Divers., 31, 57-60 (2011).

Daunton, C., S. Kothari,L. Smith and D. Steele:AHistory of materials and practices for wound management. Woud. Prac. Reser., 20, 174186 (2012).

Dolashka, P., V. Moshtanska, V. Borisova, A. Dolashki, S. Stevanovic, T. Dimanov, W. Voelter: Antimicrobial Proline-Rich Peptides from the Haemolymph of Snail. Peptides. 05, 0196-9781. (2011).

Dutta, P. K., J. Dutta and V. S. Tripathi: Chitin and chitosan: Chemistry, properties and applicatons. J. Sci. Indus. Res., 63, 02-31 (2004).

Ferraz, M.P., F.J. Monteiro and C.M. Manuel: Hydroxyapatite nanoparticles: A review of preparation methodologies. J. Appl. 
Biomat. Biomech., 2, 74-80 (2004).

Jayakumar, R., M. Prabaharan, P. T. Sutheesh Kumar, S. V. Nair and H. Tamura: Biomaterials based on chitin and chitosan in wound dressing applications. Biotechnol. Adv., 29, 322-337 (2011)

José C. V. J., D. R. Ribeaux, C. A. A. da Silva and G. M. De CamposTakaki: Physicochemical and Antibacterial Properties of Chitosan Extracted from Waste Shrimp Shells. Int. J. Microbiol., 4, 1-7, (2016).

Juknius, T., M. Ruzauskas, T. Tamulevicius, R. Siugzdiniene, I. Juknirne, A. Vasiliauskas, A. Jurkeviciute and S. Tamulevicius: Antimicrobial properties of diamond-like carbonlsilver nanocomposite thin films deposited on textiles: Towards smart bandages. Material., 9, 371$376(2016)$.

Kaewboonruang, S., N. Phatrabuddha, P. Sawangvong and S. Pitaksanurat: Comparative studies on the extraction of chitinchitosan from Golden Apple Snail shells at the control field. J. Poly. Text. Engg. 3, 34-41 (2016).

Khandelwal, H. and S. Prakash: Synthesis and characterization of hydroxyapatite powder by eggshell. J. Miner. Mater. Characteri. Engine., 4, 119-126 (2016).

Laonapakul, T.: Synthesis of hydroxyapatite from biogenic wastes. Kku.
Enginee. J., 42, 269-275(2015)

Salehzadeh, A., P. Tavacol and H. Mahjub: Bacterial, fungal and parasitic contamination of cockroaches in public hospitals of Hamadan, Iran. J. Vect. Borne. Dis., 44, 105 -110 (2007).

Shakeel, A. and I. Saiqa: Chitosan based dressing for wound care. Immuno chem Immuno pathol., 2, 16-25 (2015).

Ueno, H., T. Mori and T. Fujinaga: Topical formulations and wound healing applications of chitosan. Adv. Drug Deliv. Rev., 52, 105 $115(2001)$.

Vanitha, M.R. Kuppusamy, T.M. Sridhar, R. Sureshkumar and N. Mahalakshmi: Synthesis, characterization of nano-hydroxy apatite from white snail shells and removal of methylene blue. Int. J. Innov. Res. Advan. Engine., 4, 82-86, (2017).

Wang, T., Y. Zheng, Y. Shen, Y. Shi, F. Li, C. Su and L. Zhao: Chitosan nanoparticles loaded hydrogels promote skin wound healing through the modulation of reactive oxygen species. Artifi. Cells. Nanomed. Biotechnol., 46, 138-149 (2018).

Wanule, D., J. V. Balkhande, P.U Ratnakar, A. N. Kulkarni and C.S.Bhowate: Extraction and FTIR analysis of chitosan from American Cockroach, Periplanata americana. Int. J. Engg. Sci. Inno. Technol., 3, 299-304, (2014). 